\title{
Evaluation of Ertapenem use with Impact Assessment on Extended-Spectrum Beta-Lactamases (ESBL) Production and Gram-Negative resistance in Singapore General Hospital (SGH)
}

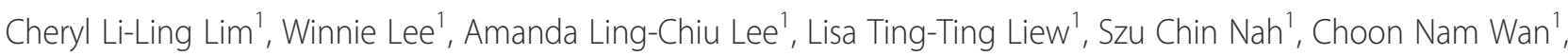
Maciej Piotr Chlebicki ${ }^{2}$ and Andrea Lay-Hoon Kwa ${ }^{*}$

\begin{abstract}
Background: Ertapenem (preferred choice for ESBL-producing organisms) use exhibited an increasing trend from 2006 to 2008. As extensive use of ertapenem might induce the mutation of resistant bacteria strains to ertapenem, we aimed to assess the appropriateness and impact of ertapenem-use, on ESBL production, the trends of gram-negative bacterial resistance and on the utilization of other antibiotics in our institution.

Methods: Inpatients who received a dose of ertapenem during 1 January 2006 to 31 December 2008, were reviewed. Pertinent patient clinical data was extracted from the pharmacy databases and assessed for appropriateness based on dose and indication. Relevant data from Network for Antimicrobial Resistance Surveillance (Singapore) (NARSS) was extracted, to cross-correlate with ertapenem via time series to assess its impact on hospital epidemiology, trends of gram-negative resistance and consumption of other antibiotics from 2006 to mid-2010.
\end{abstract}

Results: 906 cases were reviewed. Ertapenem therapy was appropriate in $72.4 \%$ (93.7\% success rate). CNS adverse events were noted in 3.2\%. Readmission rate (30-day) due to re-infection (same pathogen) was 5.5\%. Fifty cases had cultures growing Pseudomonas aeruginosa within 30 days of ertapenem initiation, with 25 cases growing carbapenem-resistant Pseudomonas aeruginosa.

Ertapenem use increased from 0.45 DDD/100 patient days in 2006 to 1.2 DDD/100 patient days in mid-2010. Overall, the increasing trend of ertapenem consumption correlated with 1) increasing incidence-densities of ciprofloxacin-resistant/cephalosporin-resistant $E$. coli at zero time lag; 2 ) increasing incidence-densities of ertapenem-resistant Escherichia. coli and Klebsiella spp. at zero time lag; 3) increasing incidence-density of carbapenem-resistant Pseudomonas aeruginosa, at zero time lag.

Increasing ertapenem consumption was significantly correlated with decreasing consumption of cefepime $\left(R^{2}=0.37344\right)$ 3 months later. It was significantly correlated with a decrease in imipenem consumption $\left(R^{2}=0.31081\right)$, with no time lag but was correlated with subsequent increasing consumption of meropenem $\left(R^{2}=0.4092\right) 6$ months later.

Conclusion: Ertapenem use was appropriate. Increasing Ertapenem consumption did not result in a decreasing trend of ESBL producing enterobacteriaceae and could result in the selection for multi-drug resistant bacteria.

Keywords: Ertapenem evaluation, ESBL production, Gram-negative resistance

\footnotetext{
* Correspondence: andrea.kwa.l.h@sgh.com.sg

'Department of Pharmacy, Singapore General Hospital, Block 8 Level 2, Outram Road, Singapore 169608, Singapore

Full list of author information is available at the end of the article
} 


\section{Background}

Antibiotic options in the treatment of extended spectrum beta-lactamases (ESBL)-producing organisms are extremely limited. Carbapenems are the treatment of choice for serious infections due to ESBL producing organisms [1]. Ertapenem, which belongs to the class of carbapenems, possesses antibacterial activity against ESBL-producing enterobacteriaceae and Haemophilus species and not the nonfermenters [2]. Hence, it is the preferred carbapenem choice for infections caused by ESBL-producing organisms currently.

Ertapenem has a longer half-life than the older carbapenems, hence allowing for once-daily administration $[3,4]$. This allows the patients to utilize the convenience of Outpatient Parenteral Antibiotic Therapy (OPAT) clinic in our institution, Singapore General Hospital (SGH), which reduces length of hospitalization in our hospital. Though ertapenem is in the hospital formulary since 2006, written or verbal approval for use has to be sought with the Infectious Diseases physicians. The use of ertapenem exhibited an increasing trend from 2006 to 2008. In July 2009, this prescribing restriction was removed. Henceforth, ertapenem is approved for use by any physicians if it is culture-directed (presence of ESBL-producing enterobacteriaceae evident by 3rd generation cephalosporins resistance) or for empiric treatment of suspected ESBL infections in patient who has a history of ESBL infections in the past 90 days. Every ertapenem case will be subjected to within 24-hour concurrent feedback audit by Antimicrobial Stewardship team.

In $\mathrm{SGH}$, where extended spectrum beta-lactamases are prevalent, there is a gradual rise in the use of carbapenems, which could bring about acquired resistance. Resistance to carbapenems is due to changes in outer membrane proteins, efflux pumps and potent metalloenzymes that cause hydrolysis of carbapenems [5]. Thus, with the introduction of ertapenem into the formulary, it was thought that we could preserve the use of the other anti-pseudomonal carbapenems for infections involving Pseudomonas aeruginosa.

On the other hand, there are concerns that extensive use of ertapenem may induce the mutation of resistant bacteria strains to ertapenem and select out Pseudomonas aeruginosa $[5,6]$ strains that might have crossresistance to other anti-pseudomonal carbapenems. While some studies have shown that ertapenem use was not associated with carbapenem-resistant Pseudomonas aeruginosa, these only looked at pharmacy purchasing databases, which may not necessarily reflect actual ertapenem prescription [7-10]. Moreover, none of the studies evaluated ertapenem use and development of gram-negative bacterial resistance at patient-level.

Hence, we evaluated the appropriateness and outcomes of ertapenem use in SGH from 2006 to 2008 and assessed the impact of ertapenem on the hospital's epidemiology, especially ESBL production, trends of gramnegative bacterial resistance, and utilization of other antibiotics, from 2006 to mid-2010 (1.5 years after the evaluation of use).

\section{Methods \\ Study design}

This study was a single-centered, retrospective study which was approved by the SingHealth Institutional Review Board.

\section{Inclusion criteria and data collection}

Patients were included in the study if they received at least one dose of ertapenem in Singapore General Hospital between 1 January 2006 and 31 December 2008 and those who were solely prescribed ertapenem as OPAT were excluded. Patient demographics and treatment related data (details of ertapenem and prior antibiotic therapy, clinical and microbiological outcomes) were extracted from the hospital's medical records and pharmacy database.

\section{Appropriateness of ertapenem use}

Ertapenem use was deemed appropriate only if criteria for both indication and dose were met. The appropriate indications for ertapenem include i) definitive therapy for ESBL producing enterobacteriaceae or non-ESBL producing enterobacteriaceae in penicillin allergic patients, where susceptibility to ertapenem is confirmed and there were limited therapeutic options left, and ii) empiric ertapenem therapy for septic patients who had prior hospitalization with positive cultures for ESBL producing enterobacteriaceae within the last 90 days. Enterobacteriaceae, that are ESBL producing, are characterized by their resistance to $3^{\text {rd }}$ generation cephalosporins (e.g. ceftriaxone and ceftazidime) [11]. ESBL production was also confirmed using double-disk diffusion method [1]. Use of ertapenem for i) Pseudomonas aeruginosa and Acinetobacter spp. infections, ii) primary bacteremia and iii) central nervous system infections (CNS) are defined as being inappropriate. Ertapenem dosing was considered appropriate if dose was adjusted according to patients' renal function (calculated using the Cockcroft-Gault equation) [12], where dose has to be reduced from $1 \mathrm{~g}$ once daily to $500 \mathrm{mg}$ once daily in patients with creatinine clearance less than $30 \mathrm{ml} / \mathrm{min}$.

\section{Outcome analysis and definitions}

The clinical efficacy and safety of ertapenem use was assessed and recorded, from the first day to the last day of ertapenem therapy. Treatment outcomes of ertapenem such as i) presence of clinical improvement and ii) presence of microbiological eradication of causative organism(s) were evaluated. Clinical improvement was 
defined as normalization of blood counts, inflammatory markers, temperature and vital signs. Microbiological eradication was concluded when there was no further bacterial growth of causative organism(s). Where repeat cultures and sensitivities were not performed, eradication was presumed if the patient was discharged clinically well. Hence, treatment success was defined as i) presence of microbiological eradication (or presumed microbiological eradication) primarily, with and without clinical improvement or ii) presence of clinical improvement (followed by home discharge), if follow up cultures were absent. New infections were defined as infections with different pathogens, that occurred after ertapenem initiation; excluding those that were present prior to ertapenem therapy.

Other outcomes used to assess treatment efficacy include 30-day mortality (from first date of ertapenem use) and discharge status (death, home or nursing home). Hospital readmission and re-infection with the same causative organism within 30 days were also evaluated.

\section{Impact of ertapenem on micro-ecology and antibiotic utilization}

To assess the association of ertapenem consumption with consumption of other broad-spectrum antibiotics, ertapenem utilization over a course of 4.5 years from 2006 to mid-2010 was correlated with the consumption of selected antibiotics (ciprofloxacin, ceftriaxone, cefepime and antipseudomonal carbapenems). All utilization data, defined as defined daily dose (DDD) per 100 inpatient-days of antibiotic, which corresponds to the assumed average daily maintenance dose, was obtained from Network for Antimicrobial Resistance Surveillance (Singapore) (NARSS) [13-16]. NARSS was established in December 2005 to conduct prospective surveillance of antibiotic resistance and prescription in Singapore public hospitals, with the aim of analyzing trends over time. Antibiotic resistance and prescription data in NARSS was obtained from the hospital laboratory information systems and pharmacy prescription databases.

Ertapenem consumption over the same period of time was also compared to the incidence density of certain microorganisms per 1000 inpatient-days (PD) reported in NARSS [15-21] to evaluate impact of ertapenem consumption on gram-negative resistance trends. These microorganisms include a) ciprofloxacin-resistant Escherichia coli and Klebsiella spp., b) cephalosporin-resistant Escherichia coli and Klebsiella spp. isolates. Incidence densities of the following microorganisms were measured as number of cases per 10,000 PD: a) ertapenem-resistant Klebsiella spp. and Escherichia coli, b) carbapenem-resistant Acinetobacter baumannii, c) carbapenem-resistant Pseudomonas aeruginosa. These microorganisms were reported in NARSS because of their potential adverse impact on nosocomial infections and public health [13-16].

\section{Statistical analysis}

Univariate analysis was done to describe the patients' demographics. Time series analysis was first performed for trend of antibiotic prescription and antimicrobial resistant bacteria over time; and if significant, crosscorrelation analysis was then performed to measure the impact of ertapenem on micro-ecology and antibiotic utilization via STATA version 10 (StataCorp LP, Texas USA).

\section{Results}

\section{Patient demographics}

A total of 929 patients were treated with ertapenem between 1 January 2006 and 31 December 2008. Twentythree patients were excluded because of unavailability of case notes. Hence, only 906 patients were analyzed. The mean duration of hospital stay was $36.55 \pm 45.53$ days. The median age of cases was $63.00 \pm 15.74$ years. More than half of the patients had underlying hypertension, diabetes mellitus and/or hyperlipidemia (Figure 1).

Table 1 described the types of infections for which ertapenem was used in the study patients, and these infections may or may not be appropriate for ertapenem use. Urinary tract infection and bacteremia were the two main infections for ertapenem use (Table 1). Out of the 306 cases that were due to bacteremia, 134 cases were due to primary bacteremia while 172 cases were due to secondary bacteremia with known sources. The main culture sites were: urine (35.2\%), blood (33.8\%), wound (23.8\%), other body fluids (11.1\%) and sputum (6.2\%). Approximately 7 out of 10 cases had ESBL infections from Escherichia coli and Klebsiella spp. (Figure 2).

\section{Appropriateness}

Approximately $72.4 \%$ of the cases fulfilled the criteria for appropriate ertapenem use. While $86.0 \%$ cases $(n=779)$ were culture-directed (Table 2), $13.2 \%$ and $0.7 \%$ of cases

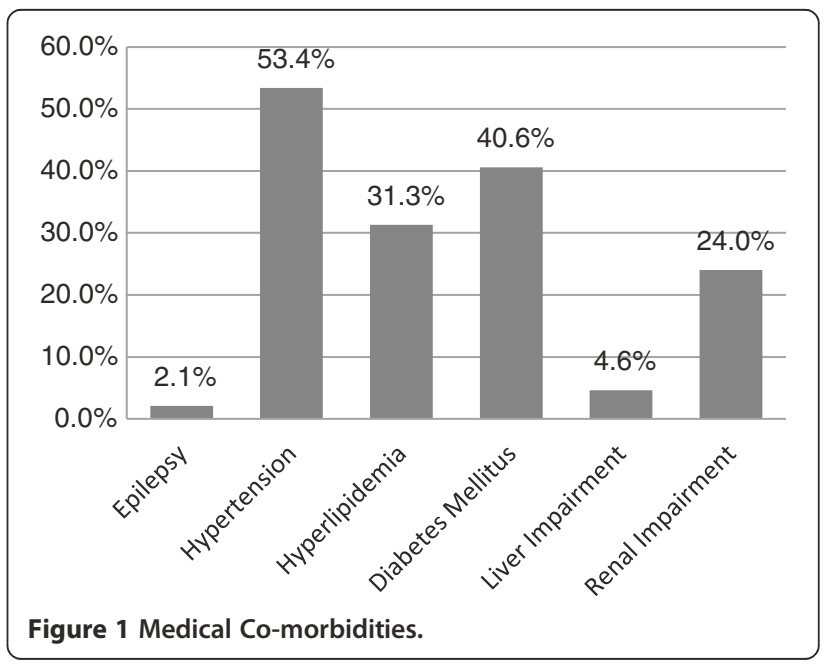


Table 1 Types of infections for which ertapenem was used

\begin{tabular}{ll}
\hline & No. of Cases (\%) \\
\hline Bacteremia & $306(33.8)$ \\
Urinary tract infection & $337(37.2)$ \\
Intra-abdominal infection & $141(15.6)$ \\
Pneumonia & $74(8.2)$ \\
Skin and soft tissue infection & $145(16.0)$ \\
Others* & $64(7.1)$ \\
\hline
\end{tabular}

*Other infections for which ertapenem was used include: line sepsis, osteomyelitis, graft infections, surgical prophylaxis and CNS infections. Patients may have had more than one type of infection for ertapenem use. These infections may or may not be appropriate.for ertapenem use.

were empirical and prophylactic, respectively. Of the culture-directed cases, $46.0 \%$ were de-escalation from broader carbapenems to ertapenem (359 out of 779 culture-directed cases). Cephalosporins (47.6\%) were the most common beta-lactam antibiotics used before ertapenem, followed by anti-pseudomonal carbapenems (39.6\%) such as imipenem and meropenem (Figure 3). As ertapenem is not indicated for surgical prophylaxis, none of the prophylaxis cases were included in the efficacy (under outcomes) analysis. The median duration of ertapenem therapy was $8(1-75)$ days.

\section{Outcomes analysis}

\section{Safety of ertapenem treatment}

Adverse events related to or suspected to be induced by ertapenem were noted in 46 cases (5.1\%), with the majority of those involving the CNS $(\mathrm{n}=29)$ (Table 3$)$. Seizures and confusion occurred in $9(1.0 \%)$ and 20 cases $(2.2 \%)$ respectively. Of the 9 cases with seizures, 4 cases $(44.4 \%)$ occurred in patients with underlying epilepsy (Table 3), where only one was inappropriately dosed. In 6 of these 9 cases (66.7\%), patients had renal impairment.
Table 2 Details of ertapenem therapy

\begin{tabular}{|c|c|}
\hline & $\begin{array}{l}\text { No. of } \\
\text { Cases (\%) }\end{array}$ \\
\hline \multicolumn{2}{|l|}{ Indication for ertapenem use } \\
\hline Empiric & $120(13.2)$ \\
\hline Prophylaxis & $7(0.7)$ \\
\hline Culture-directed & $779(86.0)$ \\
\hline \multicolumn{2}{|l|}{ Dose distribution } \\
\hline 500 milligrams once daily & $149(16.5)$ \\
\hline 1 gram every other day & $16(1.8)$ \\
\hline 1 gram once daily & $730(80.6)$ \\
\hline 1 gram stat & $6(0.6)$ \\
\hline Others^ & $5(0.5)$ \\
\hline Median duration of therapy (days) (range) & $8(1-75)$ \\
\hline Prescribed as outpatient antibiotic therapy (OPAT\#) & $185(20.4)$ \\
\hline \multicolumn{2}{|l|}{ Other antibiotic use in the same admission } \\
\hline Concurrent antibiotic therapy & $566(62.5)$ \\
\hline Previous antibiotic therapy & $778(85.9)$ \\
\hline Previous beta-lactam use ${ }^{*}$ & $754(83.3)$ \\
\hline
\end{tabular}

\section{Efficacy}

The number of cases which were included for efficacy analysis was 899 . Treatment success was observed in 842 cases (93.7\%). Out of the 57 treatment failures, 14 cases were prescribed ertapenem for bacteremia; of which 8 and 6 cases were primary and secondary bacteremia respectively. One hundred and two cases (11.3\%) had new infections, with 58 cases $(56.9 \%)$ occurring during ertapenem therapy and 44 cases $(43.1 \%)$ occurring after discontinuation of therapy. Out of the 102 cases with new infections, 8 and 4 were

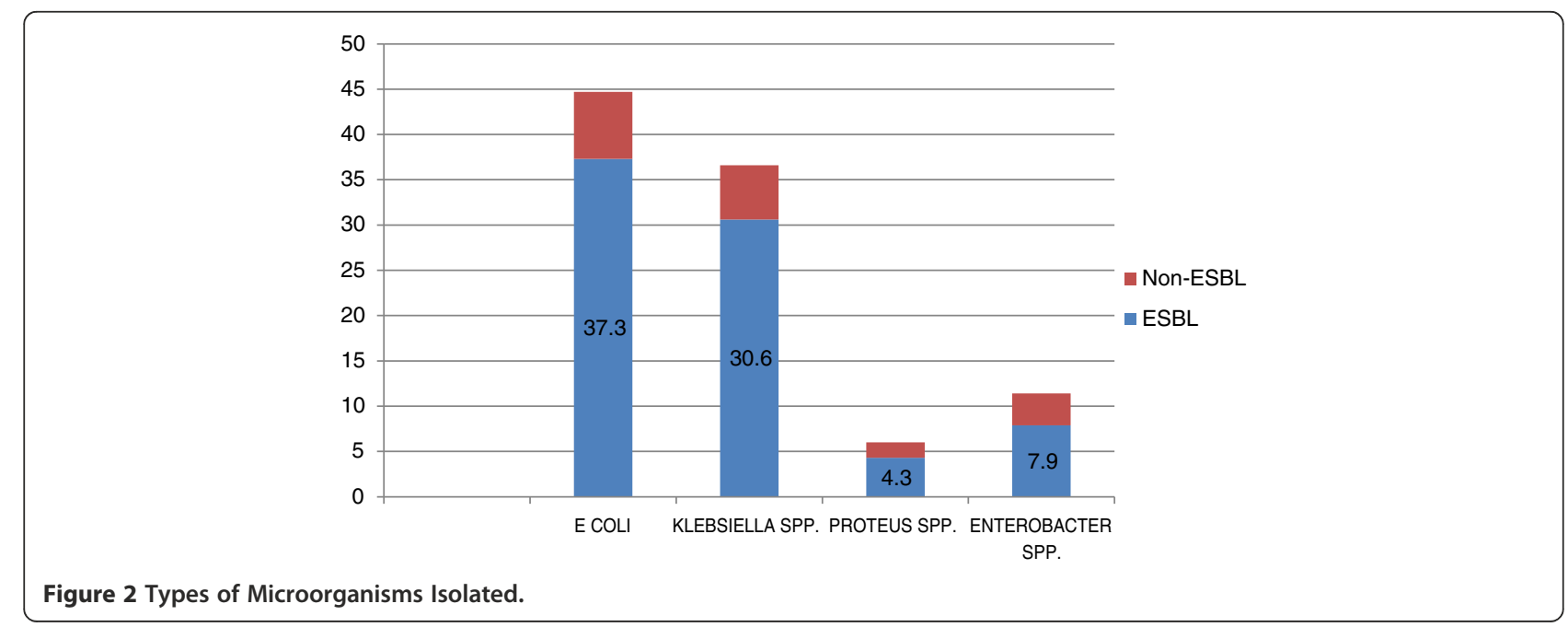




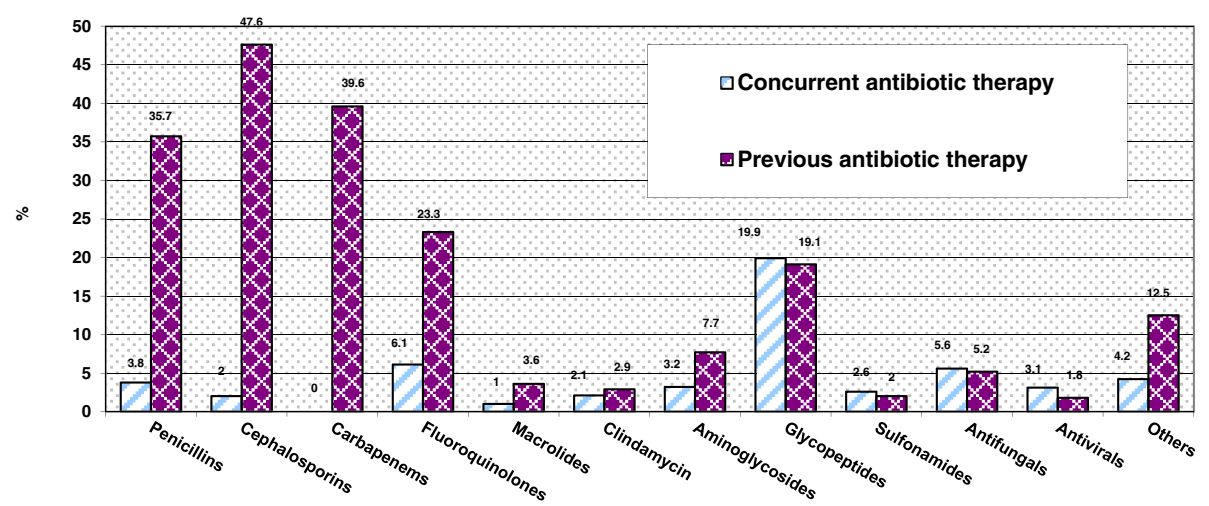

Figure 3 Previous \& Concurrent Antibiotic Therapy.

primary and secondary bacteremia respectively. Thirty-five cases with new infections were polymicrobial in nature.

\section{Mortality and re-admission}

The proportion of live-discharges was $87.9 \%(\mathrm{n}=796)$ (Table 4). Of which, 20.4\% $(\mathrm{n}=185)$ were readmitted within 30 days post-discharge with $5.5 \%(\mathrm{n}=44)$ being re-infected with the same causative microorganism. Seventy-three (36 primary and 37 secondary) out of 306 cases with bacteremia were readmitted within 30 days. Out of these 73 cases, 3 were readmitted with primary bacteremia and 4 were readmitted with secondary bacteremia. Overall mortality rate within 30 days from the first day of ertapenem treatment was $10.7 \%(n=97)$.

\section{Resistance development}

Fifty cases (5.5\%) had cultures growing Pseudomonas aeruginosa within 30 days from initiation of ertapenem therapy (Table 5) with 25 cases growing carbapenem-resistant Pseudomonas aeruginosa.

Correlation of ertapenem consumption with incidence of antibiotic-resistant bacteria

Ertapenem use increased from 0.45 DDD/100 PD in 2006 to $1.2 \mathrm{DDD} / 100 \mathrm{PD}$ in mid-2010. Cross-correlation analysis of ertapenem consumption to microorganisms with

Table 3 Adverse drug reactions

\begin{tabular}{ll}
\hline Adverse drug reaction & $\begin{array}{l}\text { No. of } \\
\text { Patients (\%) }\end{array}$ \\
\hline Seizures & $9(1.0)$ \\
Seizures occurring in those with previous history of & $4(0.4)$ \\
epilepsy & \\
Central nervous system side effects (excluding seizures) & $20(2.2)$ \\
Gastrointestinal side effects & $3(0.3)$ \\
Others* & $10(1.1)$ \\
\hline
\end{tabular}

*Other adverse drug reactions included drug fever, eosinophilia and urticaria. significant trend changes in incidence rates from 2006 to mid-2010 were performed and reported in Table 6.

Incidence-density of ciprofloxacin-resistant Escherichia coli increased from 3.2 cases per 1000 PD in 2006 to 3.7 cases per 1000 PD in mid-2010; the same increasing trend was also observed in cephalosporin-resistant Escherichia coli (1.6 to 2.0 cases per $1000 \mathrm{PD}$ ). Increasing ertapenem consumption correlated, at zero time lag, with increasing incidence-density of 1) ciprofloxacin-resistant Escherichia coli $\left(\mathrm{R}^{2}=0.2862\right)$ insignificantly and 2) cephalosporinresistant Escherichia coli $\left(\mathrm{R}^{2}=0.4442\right)$ significantly. Conversely, ciprofloxacin and ceftriaxone-resistant Klebsiella spp incidence densities decreased independently, before the increase in ertapenem consumption. There was approximately $50 \%$ decrease in incidence-density of ciprofloxacin-resistant Klebsiella spp. (2.2 to 1.1 cases per $1000 \mathrm{PD}$ ) and ceftriaxone-resistant Klebsiella spp. (2.4 to 1.5 cases per 1000 PD) from 2006 to mid-2010.

An increase in both ertapenem-resistant Escherichia coli and Klebsiella spp. was observed between 2006 and mid-2010. Increasing ertapenem consumption correlated significantly with increasing incidence-density of ertapenemresistant Escherichia coli (0.02 to 0.25 cases per 10,000 PD, $\left.\mathrm{R}^{2}=0.5103\right)$ and insignificantly with ertapenem-resistant Klebsiella spp.(0.4 to 1.0 cases per 10,000 PD, $\left.\mathrm{R}^{2}=0.2755\right)$ at zero time lag. The increasing trend of ertapenem consumption correlated with increasing incidence-density of carbapenem-resistant Pseudomonas aeruginosa, at

Table 4 Mortality and re-admission

\begin{tabular}{ll}
\hline Discharge status & Frequency (\%) \\
\hline Death & $97(10.7)$ \\
Home & $724(79.9)$ \\
Nursing home & $72(7.9)$ \\
$\begin{array}{l}\text { Hospital readmission (within 30-day of } \\
\text { discharge) }\end{array}$ & $185(20.4)$ \\
$\begin{array}{l}\text { Re-infection with the same causative } \\
\text { microorganism (in relation to hospital re-admission) }\end{array}$ & $44(5.5)$ \\
\hline
\end{tabular}


Table 5 Microorganisms isolated within $\mathbf{3 0}$ days from the start date of ertapenem therapy

\begin{tabular}{lll}
\hline Microorganism* & $\begin{array}{l}\text { Infection with new microorganism } \\
\text { within 30 days of first date of } \\
\text { ertapenem use (\%) }\end{array}$ & $\begin{array}{l}\text { Carbapenem } \\
\text { resistance (\%) }\end{array}$ \\
\hline Pseudomonas aeruginosa & $50(5.5)$ & $25(2.8)$ \\
Stenotrophomonas maltophila & $21(2.3)$ & $21(2.3)^{* *}$ \\
Acinetobacter baumannii & $27(3.0)$ & $20(2.2)$ \\
Enterobacteriaceae (such as Klebsiella spp., E. coli, Proteus spp.) with ESBL production & $41(4.5)$ & $8(0.9)$ \\
\hline
\end{tabular}

*More than one microorganism was isolated in some cases within 30 days from initiation of ertapenem therapy.

${ }^{*}$ Carbapenem sensitivity testing was not performed. However, Stenotrophomonas maltophila is intrinsically resistant to carbapenems.

increasing statistical trend ( 0.25 to 0.35 cases per 10,000 $\left.\mathrm{PD}, \mathrm{R}^{2}=0.3190\right)$, at zero time lag (Figure 4$)$.

\section{Correlation of ertapenem consumption with consumption of other antibiotics}

Ciprofloxacin use slightly increased from 1.17 DDD/100 PD in 2006 to 1.3 DDD/100 PD in mid-2010. Similarly, ceftriaxone use increased from 5.61 DDD/100 PD in 2006 to $12.5 \mathrm{DDD} / 100 \mathrm{PD}$ in mid-2010. The increasing consumption of ciprofloxacin $\left(\mathrm{R}^{2}=0.10093\right)$ and ceftriaxone $\left(R^{2}=0.22043\right)$ before, was insignificantly correlated with subsequent increasing consumption of ertapenem (Table 7). However, the increasing ertapenem consumption was correlated with subsequent decreasing consumption of cefepime 3 months later at increasing statistical trend, from 5.4 DDD/100 PD in 2006 to $4.7 \mathrm{DDD} / 100$ $\mathrm{PD}\left(\mathrm{R}^{2}=0.37344\right)$. Meropenem use increased from 2.0 DDD/100 PD in 2006 to 3.2 DDD/100 PD in mid-2010, but the converse was true for imipenem, where its use decreased from 1.8 DDD/100 PD to 0.7 DDD/100 PD in mid-2010 (Figure 5). The increase in ertapenem consumption was correlated with a decrease in imipenem consumption significantly $\left(R^{2}=0.31081\right)$, with no time lag and was correlated with subsequent increasing consumption of meropenem $\left(R^{2}=0.4092\right) 6$ months later.

\section{Discussion}

We had evaluated the appropriateness and outcomes of ertapenem use in SGH from 2006 to 2008 and assessed the impact of ertapenem on the institution's ESBL production, and trends of gram-negative bacterial resistance, and utilization of other antibiotics from 2006 to mid-2010 (4.5 years total and 1.5 years after the evaluation of use). The rate of appropriateness of ertapenem use was high. Most of our ertapenem use was culture-directed. As cephalosporins were the most common antibiotics that were administered prior to ertapenem (47.6\%), escalation from cephalosporins to ertapenem suggested that ESBL microorganisms (likely mainly ESBL producing E. coli in our institution) could have been selected out with its widespread use, since the increased prevalence of ESBL infections could result from an increased consumption of 3rd generation cephalosporins [22].

While ertapenem treatment success rates were high in SGH, seizures occurred in $1.0 \%$ of the patients $(n=9)$, which was higher than the $0.5 \%$ reported in post marketing surveillance reports [3]. This could be due to the existing concurrent medical conditions of these patients, whereby 4 out of 9 patients (44.4\%) had pre-existing epilepsy history. Two patients had a history of multiple cerebral vascular accidents; another was concurrently on morphine, which may potentiate seizures [23]; and one had a recent seizure episode that may be secondary to hypoglycemia. In addition, two-third of these patients had renal impairment, which suggested that seizures may be potentiated by drug accumulation due to decreased clearance.

Primary bacteremia is an inappropriate indication for ertapenem use in our study, due to limited evidence. Secondary bacteremia from an infective source, however, is an

Table 6 Correlation of ertapenem consumption with incidence of antibiotic-resistant bacteria

\begin{tabular}{|c|c|c|c|c|}
\hline & Correlation Coefficient* & $\mathrm{R}^{2^{*}}$ & P-values* & Time Lag \\
\hline Ciprofloxacin-resistant E. coli & 0.5349 & 0.2862 & 0.111 & Zero \\
\hline Cephalosporin-resistant E. coli & 0.6665 & 0.4442 & 0.035 & Zero \\
\hline Ciprofloxacin-resistant Klebsiella spp. & -0.4393 & 0.0393 & 0.583 & -1 year \\
\hline Cephalosporin-resistant Klebsiella spp. & -0.6752 & 0.3514 & 0.071 & -6 months \\
\hline Ertapenem-resistant E. coli & 0.7144 & 0.5103 & 0.020 & Zero \\
\hline Ertapenem-resistant Klebsiella spp. & 0.5249 & 0.2755 & 0.119 & Zero \\
\hline Carbapenem-resistant Acinetobacter baumannii & -0.6485 & 0.0911 & 0.397 & -1 year \\
\hline Carbapenem-resistant Pseudomonoas aeruginosa & 0.5648 & 0.3190 & 0.089 & Zero \\
\hline
\end{tabular}

*Correlation coefficient, $\mathrm{R}^{2}$ and P-values are obtained from cross-correlation analysis. 


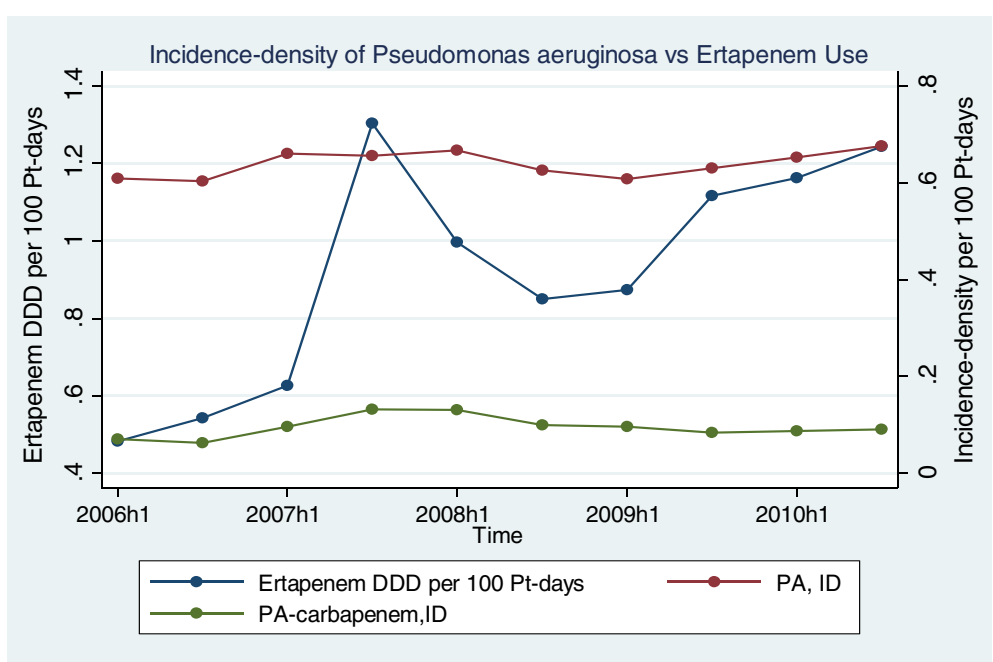

Figure 4 Ertapenem Consumption against Pseudomonas aeruginosa.

appropriate indication. In our study, out of 172 secondary bacteremia cases, 154 cases (89.5\%) had microbiological eradication. Mortality rate in these 172 cases was $13.4 \%$ (23 cases), where 6 died of other causes, and 5 others died because of concurrent infections, for example disseminated methicillin-resistant Staphylococcus aureus infection. Four secondary bacteremia cases treated with ertapenem were readmitted within 30 days with the same microorganism isolated; with 3 cases having poor source control previously, and 1 case was treated with only 5 days of ertapenem for ESBL $E$. coli bacteremia and subsequently oralized to ciprofloxacin despite intermediate resistance of $E$. coli to ciprofloxacin. This could have explained for the recurrence of bacteremia.

Evidence of ertapenem efficacy in treatment of ESBL gram-negative bacteremia has been scarce so far, with only 2 retrospective studies showing favourable clinical response in treatment of both primary and secondary bacteremia $[24,25]$. When compared with antipseudomonal carbapenems, treatment with ertapenem for gram-negative primary bacteremia led to equivalent mortality rates and bacteriological eradication [26]. It was observed in our study that out of 57 treatment failure cases, ertapenem was used for bacteremia in 14 cases; of which, 8 were primary bacteremia cases. For patients with secondary bacteremia in our study, treatment outcomes were favourable, with a high microbiological eradication rate $(89.5 \%)$ and a low mortality rate (13.4\%), thereby supporting the use of ertapenem in secondary bacteremia.

There is a concern that extensive first-line use of ertapenem will select multi-drug resistant pathogens with cross-resistance to other carbapenems $[5,6]$. Based on our study results, increasing consumption of ertapenem correlated with subsequent increasing incidences of ertapenem-resistant Escherichia coli $\left(\mathrm{R}^{2}=0.5103\right)$ significantly and carbapenem-resistant Pseudomonas aeruginosa at increasing statistical trend $\left(\mathrm{R}^{2}=0.3190\right)$. This macroscopic level of observation was also validated by our observation from the detailed evaluation of 906 cases at microscopic level. While the emergence of carbapenem-resistant Pseudomonas aeruginosa might be due to inducible resistance after exposure to ertapenem, the emergence of ertapenem-resistant Escherichia coli and Klebsiella spp. is likely to be due to the selection of an ertapenem-resistant subpopulation (ESBLs with membrane porin loss) that already pre-exist among the ertapenem susceptible population [27]. This differed from

Table 7 Correlation of ertapenem consumption with consumption of other antibiotics

\begin{tabular}{lllll}
\hline Antibiotic & Correlation Coefficient* & $\mathbf{R}^{\mathbf{2}^{*}}$ & P-values* $^{*}$ & Time Lag \\
\hline Ciprofloxacin & 0.3177 & 0.10093 & 0.717 & -3 months \\
Ceftriaxone & 0.4695 & 0.22043 & 0.657 & -3 months \\
Cefepime & -0.6111 & 0.37344 & 0.067 & 3 months \\
Imipenem & -0.5575 & 0.31081 & 0.001 & Zero \\
Meropenem & 0.2948 & 0.4092 & 0.004 & 6 months
\end{tabular}

*Correlation coefficient, $\mathrm{R}^{2}$ and P-values are obtained from cross-correlation analysis. 


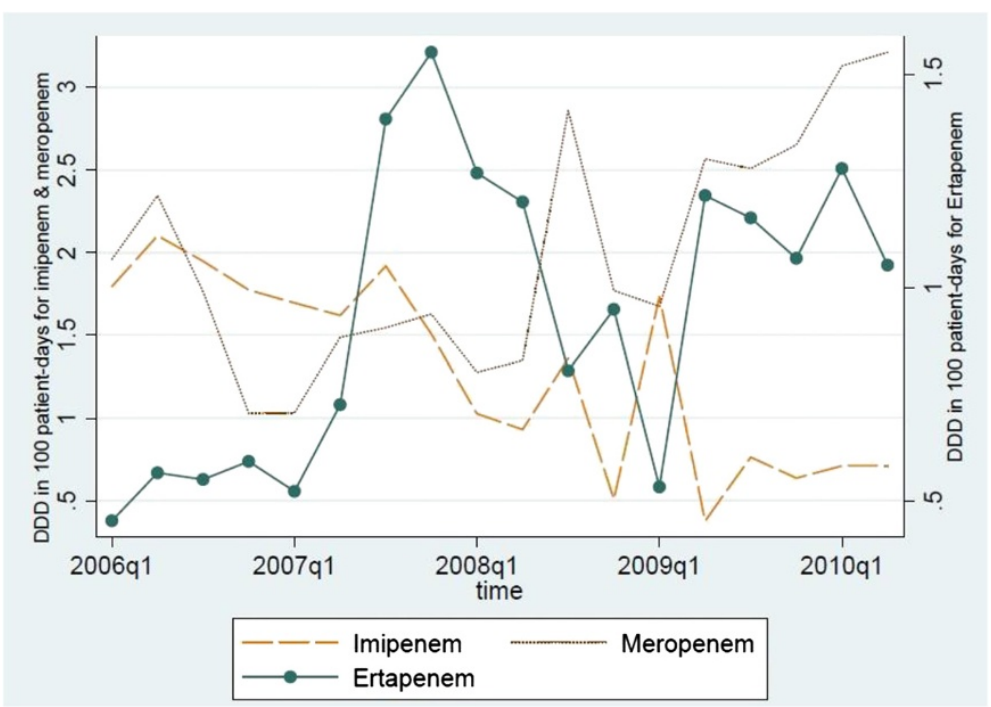

Figure 5 Ertapenem Consumption against Anti-Pseudomonal Carbapenem Consumption.

results of 3 other studies that showed no increased incidence of carbapenem-resistant Pseudomonas aeruginosa isolates after introduction of ertapenem into the hospital formularies $[9,10,28]$. In our retrospective study, a small proportion of patients developed carbapenem-resistant Pseudomonas aeruginosa (2.8\%), Acinetobacter baumannii (2.2\%), and ESBL enterobacteriaceae $(0.9 \%)$ within 30 days of ertapenem therapy initiation. Only data within 30 days was used for evaluation of infection recurrences in our study to prevent or reduce the impact of bacterial strains import from the community and other healthcare centres.

It was noted that the mean duration of hospital stay was $36.55 \pm 45.53$ days in our retrospective study. Out of 906 patients, 40 patients (4.4\%) were on ertapenem for treatment of osteomyelitis or skin abscesses, with duration of ertapenem ranging from 28 to 42 days. Twenty-eight patients (3.1\%) were treated for intraabdominal abscesses, with a median duration of 24 days. The long hospital stay may have been a risk factor in the rates of carbapenem-resistant Pseudomonas aerginosa and Acinetobacter baumannii observed, as also reported by Falagas et al [29]. However, most patients were initiated on ertapenem early during hospital stay, therefore ruling out this confounding factor.

While ciprofloxacin and ceftriaxone resistance rates in Escherichia coli continued to increase, ciprofloxacin and ceftriaxone resistant Klebsiella spp. incidence densities surprisingly decreased independently, before the increase in ertapenem consumption. Hence, the increase in ertapenem consumption could be attributed to an increase in cephalosporin and ciprofloxacin resistant Escherichia coli mainly. An increase in incidence density of community extended-spectrum beta-lactamase (ESBL)-producing and quinolone-resistant Escherichia coli isolates may have contributed to this phenomenon. Pada and co-workers had found that up to $12 \%$ of more than 1,000 emergency department attendees without previous health care association at hospital 2 in 2007 were colonized with ESBLpositive enterobacteriaceae-the vast majority of which were Escherichia coli [30]. This was because the extensive use of ciprofloxacin in community acquired infections had probably led to a continual increase in incidences of ESBLs colonization in the community.

The increasing consumption of ertapenem correlated with subsequent increasing consumption of meropenem, which may be due to selection for Pseudomonas aeruginosa with ertapenem use. However, increasing consumption of ertapenem correlated with decreasing consumption of imipenem. This could be due to physician preference for meropenem in view of relatively lower incidence of seizures with meropenem as compared to imipenem [31-33]. The consumption of cefepime, in the treatment of ESBLs, was gradually replaced by ertapenem in our institution as there were high incidences of CTX-M producing Klebsiella spp. and Escherichia coli [34] which could have high minimum inhibitory concentrations to cefepime [35-37].

There are several limitations to this work. One of the main limitations of the study was the retrospective nature of the study. Limitations inherent to retrospective review include a dependency on previously recorded data, which may be limited by systematic or recorder bias. Since data from NARSS [15-21] is extracted primarily from the hospital microbiology and pharmacy databases, it is not possible to distinguish between colonization and infection except when there are blood culture results (i.e. "bacteremia"). In addition, the study period of 4.5 years may be too short to evaluate true impact of 
ertapenem on local epidemiology. Nevertheless, it still allows the determination of any broad association between ertapenem consumption and antibiotic resistance.

In this study, only SGH data on antibiotic prescription, including ertapenem, and antimicrobial resistance trends were analyzed, therefore conclusions may not be generalized to other hospitals or community. Cross-correlation analysis of ertapenem consumption and antimicrobial resistance trends was performed without adjusting for possible confounding factors, such as consumption of other antibiotics like meropenem and length of hospitalization. Infection control measures over the same period were also not assessed, which may have been a contributing factor to transmission of drug-resistant microorganisms.

\section{Conclusions}

In conclusion, ertapenem was used appropriately in SGH, in terms of dose and indication and had resulted in a high successful treatment rate. Increasing ertapenem consumption ESBL-producing may result in the selection for multi-drug resistant bacteria, like carbapenem-resistant Pseudomonas aeruginosa. Hence, it would be prudent to judiciously use ertapenem for ESBL-producing microorganisms, with adequate dosage.

\section{Ethical approval}

The Singhealth Centralized Institutional Review Board (approval number CRIB 2010/031/D) had approved this study.

\section{Competing interest}

Kwa AL has received funding for research from Janssen-Cilag, Pfizer and Merck Sharp \& Dohme (I.A.) Corp.

\section{Authors' contributions}

LC extracted the data, carried out the data collection and analysis for both parts of the paper and drafted the manuscript. LA, LL, NSC and WCM carried out collection and analysis of data for the first part of the paper. LW and AK designed and carried out the statistical analysis. CP and AK conceived of the study and AK helped draft the manuscript. All authors read and approved the final manuscript.

\section{Acknowledgements}

This is an investigator-initiated study funded by Merck Sharp \& Dohme (I.A.) Corp.

\section{Author details}

'Department of Pharmacy, Singapore General Hospital, Block 8 Level 2, Outram Road, Singapore 169608, Singapore. ${ }^{2}$ Department of Infectious Diseases, Singapore General Hospital, Academia Level 3, 20 College Road, Singapore 169865, Singapore.

Received: 12 June 2013 Accepted: 22 October 2013

Published: 6 November 2013

\section{References}

1. Paterson DL, Bonomo RA: Extended-spectrum Beta-lactamases: A Clinical Update. Clin Microbiol Rev 2005, 18(4):657-86.

2. Shah PM, Isaac RD: Ertapenem, The First of A New Group of Carbapenems. Journal of Antimicrobial Chemotherapy 2003, 52:538-542.

3. Invanz [package insert]. Whitehouse Station, N.J: Merck \& Co., Inc; 2009. http://www.merck.com/product/usa/pi_circulars/i/invanz/invanz_pi.pdf. Accessed June 1, 2013.
4. Nix DE, Majumdar AK, DiNubile MJ: Pharmacokinetics and Pharmacodynamics of Ertapenem: An Overview for Clinicians. Journal of Antimicrobial Chemotherapy 2004, 53(S2):ii23-ii28.

5. Livermore DM, Mushtaq S, Warner M: Selectivity of Ertapenem for Pseudomonas aeruginosa Mutants Cross-resistant to Other Carbapenems. Journal of Antimicrobial Chemotherapy 2005, 55:306-311.

6. Maltezou HC: Metallo- $\beta$-lactamases in Gram-Negative Bacteria: Introducing The Era of Pan-Resistance. Int J Antimicrob Agents 2009, 33(405):1-40. e7.

7. Goff DA, Mangino JE: Ertapenem: No Effect on Aerobic Gram-Negative Susceptibilities to Imipenem. Journal of Infection 2008, 57:123-127.

8. McDougall DAJ, Mortan AP, Playford EG: Association of Ertapenem and Antipseudomonal Carbapenem Usage and Carbapenem Resistance in Pseudomonas aeruginosa Among 12 Hospitals in Queensland, Australia. Journal of Antimicrobial Chemotherapy 2013, 68:457-460.

9. Eagye KJ: MPH, Nicolau D P: Absence of Association Between Use of Ertapenem and Change in Antipseudomonal Carbapenem Susceptibility Rates In 25 Hospitals. Infect Control Hosp Epidemiol 2010, 31(5):485-90.

10. Goldstein EJC, Citron DM, Peraino V, Elgourt T, Meibohm AR, Lu S: Introduction of Ertapenem Into a Hospital Formulary: Effect On Antimicrobial Usage and Improved In Vitro Susceptibility of Pseudomonas aeruginosa. Antimicrob Agents Chemother 2009, 53(12):5122-5126.

11. Emery $C L$, Weymouth $L A$ : Detection and Clinical Significance of Extendedspectrum Beta-lactamases in A Tertiary-care Medical Center. J Clin Microbiol 1997, 35(8):2061-2067.

12. Cockcroft DW, Gault MH: Prediction of Creatinine Clearance from Serum Creatinine. Nephron 1976, 16(1):31-41.

13. Filius PMG, Liem TBY, van der Linden PD, Janknegt $R$, Natsch $S$, Vulto AG Verbrugh HA: An additional measure for quantifying antibiotic use in hospitals. Journal of Antimicrobial Chemotherapy 2005, 55:805-808.

14. WHO Collaborating Centre for Drug Statistics Methodology (Norway): Guidelines For ATC Classification and DDD Assignment 2010. Oslo, Norway: WHO Collaborating Centre; 2010.

15. Koh TH, Jureen R, Tan TY, Hsu LY, Krishnan P, Tee N: Network for Antimicrobial Resistance Surveillance (Singapore) Version 1.0. Antimicrobial Resistance Report: July to December 2008. 2008. Unpublished Data.

16. Koh TH, Jureen R, Tan TY, Chiew YF, Hsu LY, Krishnan P, Tee N: Network for Antimicrobial Resistance Surveillance (Singapore) Version 1.2. Antimicrobial Utilization Report. 2009. July to December 2009. Unpublished Data.

17. Koh TH, Tan TY, Jureen R, Hsu LY, Krishnan P, Tee N, Chan J: Network for Antimicrobial Resistance Surveillance (Singapore) 2011. Carbapenem-Resistant Pseudomonas Aeruginosa. 2011. 2006-2010. Unpublished Data.

18. Koh TH, Tan TY, Jureen R, Hsu LY, Krishnan P, Tee N, Chan J: Network for Antimicrobial Resistance Surveillance (Singapore) 2011. Carbapenem-Resistant Acinetobacter. 2011. 2006-2010. Unpublished Data.

19. Koh TH, Tan TY, Jureen R, Hsu LY, Krishnan P, Tee N, Chan J: Network for Antimicrobial Resistance Surveillance (Singapore). Klebsiella pneumoniae 2011. 2011. 2006-2010. Unpublished Data.

20. Koh TH, Tan TY, Jureen R, Hsu LY, Krishnan P, Tee N, Chan J: Network for Antimicrobial Resistance Surveillance (Singapore). Escherichia coli 2011. 2011. 2006-2010. Unpublished Data.

21. Koh TH, Tan TY, Jureen R, Hsu LY, Krishnan P, Tee N, Chan J: Network for Antimicrobial Resistance Surveillance (Singapore). Klebsiella pneumoniae: 2006-2010. Unpublished Data.

22. Urbánek K, Kolár M, Lovecková Y, Strojil J, Santavá L: Influence of ThirdGeneration Cephalosporin Utilization on the Occurrence of ESBL-positive Klebsiella pneumoniae Strains. J Clin Pharm Ther 2007, 32(4):403-8.

23. Lacy CF, Armstrong LL, Goldman MP, Lance LL: Drug Information Handbook. 20th edition. Hudson, Ohio: Lexi-Comp, Inc; 2011:623-4.

24. Lye DC, Wijaya L, Chan J, Teng CP, Leo YS: Ertapenem for Treatment of Extended-Spectrum Beta-lactamase-producing Multidrug-resistant Gram-Negative Bacteremia. Annals Academy of Medicine 2008, 37:831-4.

25. Lee NY, Huang WH, Tsui KC, Hsueh PR, Ko WC: Carbapenem Therapy for Bacteremia Due to Extended-spectrum Beta-lactamase-producing Escherichia coli or Klebsiella pneumoniae. Diagnostic Microbiology \& Infectious Disease 2011, 70(1):150-3.

26. Wu UI, Chen WC, Yang CS, Wang JL, Hu FC, Chang SC, Chen YC: Ertapenem in the Treatment of Bacteremia Caused by Extended-spectrum Beta-lactamase-producing Escherichia coli: A Propensity Score Analysis. Int J Infect Dis 2012, 16(1):47-52. 
27. Leavitt A, Chmelnitsky I, Colodner R, Ofek I, Carmeli Y, Navon-Venezia S: Ertapenem Resistance Among Extended-Spectrum-Beta-LactamaseProducing Klebsiella pneumoniae. J Clin Microbiol 2009, 47(4):969-74

28. Lima ALLM, Oliveria PRD, Paula AP, Zumiotti AV: Influence of Ertapenem Administration On the Incidence of Carbapenem-Resistant Pseudomonas Aeruginosa. Brazilian Journal of Infectious Diseases 2008, 12(2):105-106.

29. Falagas ME, Kopterides P: Risk Factors For the Isolation of Multi-DrugResistant Acinetobacter baumannii and Pseudomonas aeruginosa: A Systematic Review of the Literature. Journal of Hospital Infection 2006, 64:7-15.

30. Pada S, Lye DC, Krishnan P, Leo YS: Prevalence and Predictors of Methicillinresistant Staphylococcus aureus (MRSA) and Extended-spectrum Betalactamase (ESBL) Gram-negative Bacteria at Hospital Presentation in Singapore, abstr. 69.009. Kuala Lumpur, Malaysia: 13th International Congress on Infectious Dieases 2008; 2008

31. Collazose J, Asensi V, Cartona JA, JFierer D, EBustillo C, RodriguezGuardadoa A, Suarez-Zarracinaa T, Asensib JM, Saavedraa A, Martin-Canala G: Meropenem Monotherapy is as Effective as and Safer than Imipenem to Treat Brain Abscesses. Int J Antimicrob Agents 2010, 35(3):301-4.

32. Peter L: Safety Profile of Meropenem: An Updated Review of Over 6000 Patients Treated with Meropenem. Drug Saf 2007, 30(8):657-668.

33. Claudine MB, Katherine A, Lyseng-Williamson, Susan JK: Meropenem: A Review of its Use in the Treatment of Serious Bacterial Infections. Drugs 2008, 68(6):803-838.

34. Tan TY, Ng LS, He J, Hsu LY: CTX-M and AmpC Beta-lactamases Contributing to Increased Prevalence of Ceftriaxone-resistant Escherichia coli in Changi General Hospital, Singapore. Diagn Microbiol Infect Dis 2010, 66(2):210-3.

35. Bauernfeind $A$, Casellas JM, Goldberg M, Holley $M$, Junwirth $R$, Mangold $P$, Rohnisch T, Schweighart S, Wilhelm R: A New Plasmidic Cefotaximase from Patients Infected with Salmonella typhimurium. Infection 1992, 20:158-63.

36. Palucha A, Mikiewicz B, Hryniewicz W, Gniadkowski M: Concurrent Outbreaks of Extended-spectrum Beta-lactamase-producing Organisms of the Family Enterobacteriaceae in a Warsaw Hospital. Journal of Antimicrobial Chemotherapy 1999, 44:489-99.

37. Szabo D, Filetoth Z, Szentandrassy J, Nemedi M, Toth E, Jeney C, Kispal G, Rozgonyi F: Molecular Epidemiology of A Cluster of Cases due to Klebsiella pneumoniae producing SHV-5 Extended-spectrum B-lactamase in the Premature Intensive Care Unit of a Hungarian Hospital. J Clin Microbiol 1999, 37:4167-9.

doi:10.1186/1471-2334-13-523

Cite this article as: Lim et al: Evaluation of Ertapenem use with Impact Assessment on Extended-Spectrum Beta-Lactamases (ESBL)

Production and Gram-Negative resistance in Singapore General Hospital (SGH). BMC Infectious Diseases 2013 13:523.

\section{Submit your next manuscript to BioMed Central and take full advantage of:}

- Convenient online submission

- Thorough peer review

- No space constraints or color figure charges

- Immediate publication on acceptance

- Inclusion in PubMed, CAS, Scopus and Google Scholar

- Research which is freely available for redistribution 